\title{
INDEPENDÊNCIA FUNCIONAL E TOLERÂNCIA AO EXERCÍCIO FÍSICO EM PORTADORES DE DPOC
}

\author{
Natacha Angélica da Fonseca Miranda ${ }^{l}$ \\ Diogo Fanfa Bordin ${ }^{l}$ \\ Camila da Cunha Niedermeyer ${ }^{l}$ \\ Gustavo Jungblut Kniphoff ${ }^{1}$ \\ Juliano Rodrigues Adolfo ${ }^{2}$ \\ Dannuey Machado Cardoso ${ }^{3}$ \\ Dulciane Nunes Paiva ${ }^{3}$ \\ Andréa Lúcia Gonçalves da Silva
}

\begin{abstract}
RESUMO
A Doença Pulmonar Obstrutiva Crônica (DPOC) é caracterizada por uma limitação progressiva ao fluxo aéreo, de origem inflamatória e com repercussões multissistêmicas, que se traduz como dispneia, intolerância ao exercício e baixa capacidade funcional. Este estudo em andamento objetivou quantificar a independência funcional e a tolerância ao exercício físico em portadores de DPOC ingressantes no Programa de Reabilitação Pulmonar (PRP) do Hospital Santa Cruz. Estudo de delineamento transversal, do tipo estudo de casos, avaliou-se a independência funcional através do questionário de Medida de Independência Funcional (MIF) e a tolerância ao exercício, através do teste máximo de carga incremental (Shuttle Test). Foram avaliados 14 portadores de DPOC, com idade média de 66,93 $\pm 6,22$ e predominância do sexo masculino. Uma média de $122,21 \pm 6,60$ pontos no MIF foi encontrada, caracterizando-os como independência completa ou modificada. A distância média percorrida no Shuttle Test foi de 370,11 $\pm 114,53$ metros e observou-se que, quanto mais grave a DPOC, menor foi a distância percorrida pelos sujeitos. Os portadores de DPOC deste estudo são independentes funcionalmente, porém apresentam diferentes níveis de tolerância ao exercício físico incremental e este parece estar associado com a progressão da doença.
\end{abstract}

Palavras-chave: DPOC. Reabilitação. Teste de esforço.

\begin{abstract}
The Chronic Obstructive Pulmonary Disease (COPD) is characterized by progressive airflow limitation, of inflammatory origin and multisystemic effects, which translates as dyspnea, exercise intolerance and low functional capacity. This ongoing study aimed to assess the functional independence and exercise tolerance in COPD patients entering the Pulmonary Rehabilitation Program (PRP) from Hospital Santa Cruz. Of cross-sectional, case studies type, evaluated the functional independence through the Functional Independence Measure questionnaire (MIF) and exercise tolerance with the maximum test load incremental (Shuttle Test). We evaluated 14 patients with COPD, with a mean age of $66.93 \pm 6.22$ and

\footnotetext{
1 Alunos do Curso de Fisioterapia da Universidade de Santa Cruz do Sul - UNISC.< natacha@mx2.unisc.com.br>; <camila_niedermeyer@hotmail.com>; <diogofanfa@hotmail.com>; $<$ kniphoff_8@hotmail.com >

${ }^{2}$ Residente multiprofissional em Saúde pelo Hospital Santa Cruz (HSC), Santa Cruz do Sul, RS, Brasil. Educador Físico pela Pontifícia Universidade Católica do Rio Grande do Sul (PUCRS), Porto Alegre, RS, Brasil. <julianorodrigues@unisc.br>

3 Professores do Departamento de Educação Física e Saúde na Universidade de Santa Cruz do Sul- UNISC. < dannueyc@unisc.br>; <dulciane@unisc.br>; <andreag@unisc.br>
} 
predominantly male. An average of $122.21 \pm 6.60$ points in the MIF was found, characterizing them as full or modified independence. The average distance traveled in the Shuttle Test was $370.11 \pm 114.53$ meters and it was observed that how much more severe was COPD the lowest was the distance traveled by the subjects. COPD patients in this study are functionally independent, but have different levels of tolerance to incremental exercise and this seems to be associated with disease progression.

Keywords: COPD. Rehabilitation. Test effort.

\section{INTRODUÇÃO}

A compreensão sobre a fisiopatologia da Doença Pulmonar Obstrutiva Crônica (DPOC) vem se alterando, devido à grande escala de estudos e pesquisas sobre a patologia. Apesar de afetar prioritariamente a função pulmonar, a DPOC tem repercussões multissistêmicas e necessita de um tratamento integral, abrangendo a complexidade da doença (GOLD, 2014). A dispneia, um dos principais sintomas da doença, está associada à fraqueza muscular respiratória e a disfunção muscular periférica, gerando assim uma fraqueza muscular generalizada. Quando agrupados, esses sintomas interferem no condicionamento físico, levando o sujeito à intolerância ao exercício e a limitação nas atividades diárias (NUSSBAUMER-OCHSNER; RABE, 2011).

Contudo, levando em consideração que a DPOC é uma doença de caráter progressivo, é necessária a utilização de um programa de reabilitação pulmonar (PRP) que complemente o tratamento farmacológico, pois isso irá minimizar a taxa de exacerbação e os altos custos gerados por essa doença (GOLD, 2014; SPRUIT et al., 2013). O ingresso em PRP ocorre mediante a realização de uma avaliação completa e adequada, utilizando testes máximos, submáximos e questionários que avaliem a qualidade de vida e a independência funcional (SPRUIT et al., 2013; PASQUA et al., 2009).

Sendo assim, pretende-se, através deste estudo, quantificar a independência funcional e a tolerância ao exercício físico em portadores de DPOC ingressantes no programa de reabilitação pulmonar (PRP) do Hospital Santa Cruz.

\section{FUNDAMENTAÇÃO TEÓRICA}

A Doença Pulmonar Obstrutiva Crônica (DPOC) é caracterizada por uma limitação ao fluxo aéreo em decorrência da combinação de duas patologias: a bronquite crônica (pequenas vias aéreas) e o enfisema (destruição do parênquima). Outra característica 
importante desta doença é o seu aspecto progressivo, associado à inflamação crônica do sistema respiratório à inalação de partículas e gases nocivos (GOLD, 2014). Atualmente, o termo "presença de comorbidades" foi associado à definição de DPOC, devido à ocorrência de manifestações multissistêmicas e presença de outras comorbidades (SPRUIT et al., 2013). Outro fator relevante é o aumento da ocorrência da DPOC relacionado ao envelhecimento da população (NUSSBAUMER-OCHSNER; RABE, 2011).

Um dos principais agentes causadores da DPOC é a exposição ao tabaco. O tabagismo, além de causar danos diretos ao tecido pulmonar, causa outros desfechos clínicos que interferem no sistema musculoesquelético e no condicionamento físico (NUSSBAUMER-OCHSNER; RABE，2011). Dentre as alterações musculoesqueléticas, destacamos a mudança da configuração das fibras musculares que, depois de instaladas as alterações sistêmicas causadas pela DPOC, se tornam na maioria glicolíticas e atrofia muscular. Neste sistema muscular alterado, ocorre a utilização precoce do metabolismo anaeróbico, o que implica em fadiga muscular e na baixa tolerância ao exercício (SANTOS; VIEGAS, 2009). Devido às alterações fisiopatológicas, o portador de DPOC apresenta intolerância ao exercício, levando-o a sua baixa qualidade de vida (BOREL et al., 2013).

As comorbidades associadas às alterações sistêmicas na DPOC exigem a realização de uma avaliação e tratamento amplos, ou seja, um plano terapêutico que beneficie e tenha repercussão sistêmica (CARREIRO et al., 2012). Nesse sentido, a avaliação da limitação ao exercício físico e da redução da capacidade funcional deve ser realizada de forma minuciosa, permitindo a avaliação dos múltiplos fatores envolvidos, tais como: a dispneia, distúrbios na troca gasosa, disfunção muscular periférica, hiperinsuflação dinâmica e sobrecarga no trabalho ventilatório (SPRUIT et al., 2013). O resultado de tal processo de avaliação pode inclusive ser utilizado como marcador de progressão da doença (BOREL et al., 2013).

O questionário para realizar a medida da independência funcional (MIF) é uma ferramenta útil e validada para aferir a independência em níveis cognitivos e motor (PASQUA et al., 2009). Segundo Radosavljevic et al. (2014), a possível melhora da funcionalidade está associada a fatores como idade, sexo, comorbidade e raça. A DPOC é uma patologia que acomete, em maior número, pessoas com idade avançada. Dessa forma, ressalta-se que o declínio da independência funcional também está associado a este fator (FERREIRA et al., 2012).

A avaliação da tolerância ao exercício pode ser realizada através de testes máximos ou submáximos, dentre os quais se destaca o teste de carga incremental, mais conhecido por Shuttle Test. O Shuttle Test é um método simples e de fácil reprodutibilidade, considerado um 
teste máximo para esta população de doentes pelo fato de ser incremental; exige respostas fisiológicas semelhantes às obtidas em teste de exercício cardiopulmonar. A literatura indica que originalmente o teste foi criado para avaliar a capacidade funcional em portadores de DPOC e que a distância caminhada reflete como marcador do prognóstico da doença (DOURADO et al., 2013). Além de ser útil para avaliação da capacidade funcional, auxilia para realizar a prescrição de exercícios, dentro de um PRP (BOREL et al., 2013).

\section{MATERIAIS E MÉTODOS}

O presente estudo é de delineamento transversal, do tipo estudo de casos, composto por amostragem de conveniência, triada entre os portadores de DPOC participantes do PRP, em Santa Cruz do Sul - RS. A amostra utilizada pertence ao projeto de pesquisa Efeitos da Reabilitação Pulmonar sobre a capacidade cardiorrespiratória e funcional de portadores de Doença Pulmonar Obstrutiva Crônica, no município de Santa Cruz do Sul, aprovado pelo Comitê de Ética em Pesquisa da Universidade de Santa Cruz do Sul, sob protocolo de número $435.093 / 2013$.

Foram incluídos no estudo, indivíduos com idade entre 40 a 80 anos, com diagnóstico clínico de DPOC, mediante prova de função pulmonar por espirometria, e estáveis clinicamente. Em relação ao estadiamento da DPOC, após a prova de função pulmonar, a amostra foi estratificada entre grau de obstrução moderado a muito severo, conforme determinado pela GOLD (2014). Foram excluídos os sujeitos que apresentavam desordens musculoesqueléticas, sequelas neurológicas que afetassem o aparelho locomotor e déficit cognitivo, lesões cutâneas em região do pé, agudização da doença em 30 dias prévios ao estudo e diagnóstico de câncer de pulmão.

A coleta de dados foi realizada entre abril e julho de 2014, no PRP alocado no Hospital Santa Cruz, na cidade de Santa Cruz do Sul - RS. As variáveis utilizadas para esse estudo foram as relacionadas à independência funcional e tolerância ao exercício físico. A independência funcional foi avaliada através do questionário de Medida de Independência Funcional (MIF), que tem como finalidade verificar o desempenho do indivíduo para a realização de 18 tarefas referentes aos domínios motor (que abrange autocuidado, controle esfincteriano, transferências, locomoção) e cognitivo social (comunicação e cognição social). O valor atribuído a cada tarefa varia de 01 (assistência total) a 07 (independência completa), variando do valor mínimo de 18 ao máximo de 126 (TALMELLI et al., 2010). Já a tolerância ao exercício foi avaliada através do Shuttle Test, que é realizado em corredor plano, sendo o 
paciente orientado a caminhar em percurso de 10 metros, com aumentos progressivos da cadência de caminhada ditados por mídia sonora; este é finalizado quando o sujeito não consegue chegar ao final do percurso antes do bipe (DOURADO et al., 2013).

Os dados foram armazenados no banco de dados computadorizado da pesquisa. A análise estatística foi realizada pelo programa Statistical Package for Social Sciences for Windows (versão 20.0) e descrita através de métodos descritivos expressos em valores percentuais, médias e desvios-padrão.

\section{RESULTADOS E DISCUSSÃO}

Foram avaliados nesse estudo, 14 portadores de DPOC com predominância do sexo masculino ( $\mathrm{n}=09$ ), média de idade de 66,93 $\pm 6,22$ anos e índice de massa corporal (IMC) médio de $28,66 \pm 6,71 \mathrm{Kg} / \mathrm{m}^{2}$ (Tabela 1 ).

Tabela 1. Características clínicas e sociodemográficas dos portadores de DPOC.

\begin{tabular}{|c|c|}
\hline Variáveis & DPOC, $n=14(100 \%)$ \\
\hline Sexo masculino & $09(64,3)$ \\
\hline Idade (anos) & $66,93 \pm 6,22$ \\
\hline \multicolumn{2}{|l|}{ Função Pulmonar (\% predito) ${ }^{\#}$} \\
\hline CVF & $68,72 \pm 17,59$ \\
\hline $\mathrm{VEF}_{1}$ & $47,47 \pm 16,56$ \\
\hline $\mathrm{VEF}_{1} / \mathrm{CVF}$ & $68,63 \pm 18,48$ \\
\hline $\operatorname{IMC}\left(\mathrm{Kg} / \mathrm{m}^{2}\right)$ & $66,00 \pm 6,71$ \\
\hline \multicolumn{2}{|l|}{ Classificação do IMC } \\
\hline Magreza & $02(14,3)$ \\
\hline Eutrófico & $04(28,6)$ \\
\hline Obesidade & $08(57,1)$ \\
\hline \multicolumn{2}{|l|}{ Escolaridade } \\
\hline Fundamental Incompleto & $10(71,4)$ \\
\hline Fundamental Completo & $04(28,6)$ \\
\hline \multicolumn{2}{|l|}{ Etnia } \\
\hline Caucasiano & $12(85,7)$ \\
\hline Pardo & $01(7,1)$ \\
\hline Negro & $01(7,1)$ \\
\hline
\end{tabular}

A amostra estudada vem ao encontro da literatura, em que a maioria dos sujeitos acometidos pela DPOC são do sexo masculino e em idade adulta avançada (GOLD, 2014). Embora a maioria dos sujeitos, aqui avaliados, tenha apresentando um IMC elevado, sabe-se que a tendência dessa população, devido o curso da doença, é a magreza (ARAÚJO; 
HOLANDA, 2010). Há evidências de que o baixo IMC está relacionado à baixa sobrevida nesta população e está associado principalmente à fraqueza muscular diafragmática, diminuição da função pulmonar e inflamação sistêmica. No entanto, apesar de pouco elucidado, o excesso de peso ou obesidade tem sido demonstrado como fator de proteção contra a mortalidade nessa população (CAO et al., 2012).

Os sujeitos deste estudo apresentaram baixa escolaridade e, neste sentido, tendem a uma maior prevalência do tabagismo, decorrente das informações limitadas sobre a repercussão dos efeitos deste sobre o sistema respiratório e consequentemente baixa qualidade de vida (FERNANDES, 2009). A predominância da etnia caucasiana se justifica pela região onde o estudo está sendo realizado, ou seja, Santa Cruz do Sul/RS, cidade de colonização germânica.

Na tabela 2 estão descritos os resultados obtidos no questionário MIF, no Shuttle Test, bem como a estratificação deste conforme estadiamento da doença.

Tabela 2. Desempenho dos portadores de DPOC no Questionário MIF e Shuttle Test.

\begin{tabular}{ll}
\hline Variáveis & DPOC, $\mathrm{n}=14$ \\
\hline MIF & $122,21 \pm 6,60$ \\
Mínimo de pontos & 101,0 \\
Máximo de pontos & 126,0 \\
Shuttle Test & \\
Distância caminhada (m) & $370,11 \pm 114,53$ \\
\% predito em relação a sujeitos saudáveis* & $48,63 \pm 14,29$ \\
Distância mínima (m) & 188,50 \\
Distância máxima (m) & 676,34 \\
Shuttle Test estratificado por estadiamento da doença & \\
(\% predito) & \\
DPOC Moderado & $55,92 \pm 12,88$ \\
DPOC Severo & $47,61 \pm 12,95$ \\
DPOC Muito Severo & $42,16 \pm 9,10$ \\
\hline
\end{tabular}

* Probst et al., Reference values for the incremental shuttle walking test.

A pontuação média do MIF encontrada nestes sujeitos $(122,21 \pm 6,60$ pontos $)$ classifica-os em sujeitos com independência funcional completa ou independência funcional modificada em decorrência do uso de óculos para correção visual. Há relatos na literatura que associam a independência funcional em idade avançada às atividades de vida diária, mesmo que essa população não realize um exercício físico propriamente dito (FERREIRA et al., 2012). Apesar de o declínio da capacidade funcional estar associada ao envelhecimento, os indivíduos parecem manter um nível de independência completo/modificado devido à 
composição do questionário em que os domínios são verificados a partir de atividades básicas de vida diária. O comprometimento das atividades durante o envelhecimento ocorre de acordo com a sua complexidade, sendo as atividades básicas de vida diária, as últimas a serem afetadas (SPOSITO et al., 2010).

A distância média caminhada pelos portadores de DPOC no Shuttle Test foi o equivalente a 48,63 $\pm 14,29 \%$ do predito para sujeitos saudáveis nesta faixa etária. Importante ressaltar que houve grande variabilidade na distância caminhada nestes pacientes (mínimo= 188,50m; máximo $=676,34 \mathrm{~m}$ ). O resultado do nosso estudo vai ao encontro do realizado por Dias et al. (2014), em que a intolerância ao exercício pode ser avaliada através da distância percorrida e do previsto, conforme sua composição corporal, sexo, altura e idade. A literatura ainda ressalta que há correlação entre a distância percorrida nesse teste, com a qualidade de vida e a capacidade funcional, sendo que os sujeitos que percorrem uma distância reduzida possuem uma qualidade de vida inferior e os que percorrem uma maior distância possuem melhor capacidade funcional (NASCIMENTO et al., 2014). Outro fato é que a distância percorrida é um forte preditor do estado de saúde e se correlaciona a diferentes domínios em questionários de qualidade de vida, mais especificamente Short Form 36 (SF-36) e Saint George Respiratory Questionnaire (SGRQ) (DOWSON et al., 2001).

Quando analisamos o resultado do Shuttle Test (\% do predito), estratificando pelo estadiamento da DPOC, os resultados mostraram que, conforme a doença avança, o desempenho no Shuttle Test se reduz. Nossos resultados vem ao encontro do estudo de Oliveira et al. (2014), em que o trabalho realizado por sujeitos classificados em GOLD II (moderado) foi maior em relação aos GOLD III e IV (pouco severo e severo, respectivamente) na distância percorrida no Shuttle Test. Isto pode ser justificado devido às alterações sistêmicas da DPOC que se tornam mais pronunciadas com a sua progressão, principalmente disfunção muscular periférica, alteração da composição das fibras musculares e dispneia aos esforços (DIAS et al., 2014).

Ressalta-se ainda que em sujeitos que apresentam limitação crônica no fluxo aéreo, como os DPOC, o Shuttle Test torna-se um método que facilita a mensuração da capacidade funcional e, por não ter interferência de estímulo externo, acaba por desencadear a produção do limite máximo ao exercício físico.

Este estudo, ainda em andamento, apresenta limitações devido ao baixo número amostral. Sendo assim, não foi possível fazer uma análise estatística adequada já que não foram permitidas muitas inferências à população do estudo. 


\section{CONCLUSÃO}

Os portadores de DPOC deste estudo são independentes funcionalmente, porém apresentam diferentes níveis de tolerância ao exercício físico incremental e este parece estar associado com a progressão da doença.

\section{AGRADECIMENTOS}

Agradecemos a todos os pacientes, portadores de DPOC, que foram voluntários nesta pesquisa e que permitiram a construção do conhecimento exposto.

\section{SUPORTE E FINANCIAMENTO}

Universidade de Santa Cruz do Sul- UNISC, DECIT/SCTIE-MS/FAPERGS/CNPq 1264-2551/13-1 e Hospital Santa Cruz.

\section{REFERÊNCIAS}

BOREL, B. et al. Responsiveness of various exercise-testing protocols to therapeutic interventions in COPD. Pulmonary Medicine, New York, v. 2013, p. 01-12, jan. 2013.

CAO, C. et al. Body mass index and mortality in chronic obstructive pulmonary disease: a meta-analysis. PLoS One, n. 8, v. 7, p. 01-08 ago. 2012.

CARREIRO, A.; SANTOS, J.; RODRIGUES, F. Impacto das comorbilidades num programa de reabilitação respiratória em doentes com doença pulmonar obstrutiva crônica. Revista Portuguesa de Pneumologia, n. 3, v. 19, p.106-113, 2013.

DIAS, F. D. et al. Avaliação da composição corporal, capacidade funcional e função pulmonar em pacientes com Doença Pulmonar Obstrutiva Crônica. Fisioterapia e Pesquisa, São Paulo, n. 1, v. 21, p. 10-15, jan./mar. 2014.

DOURADO, V. Z. et al. Valores de referência para o teste de caminhada com carga progressiva em indivíduos saudáveis: da distância percorrida às respostas fisiológicas. Jornal Brasileiro de Pneumologia, São Paulo, n. 2, v. 39, p. 190-197, mar./abr. 2013.

DOWSON, L. J. et al. Exercise capacity predicts health status in a 1- antitrypsin deficiency. American Journal of Respiratory and Critical Care Medicine, Nova York, n. 4, v. 163, p. 936-941, mar. 2001.

FERNANDES, A. B. S. Reabilitação respiratória em DPOC - a importância da abordagem fisioterapêutica. Pulmão RJ, Rio de Janeiro, n. 1, v. 1, p. 71-78, 2009. 
FERREIRA, O. G. L. et al. Envelhecimento ativo e sua relação com a independência funcional. Texto \& Contexto - Enfermagem, Florianópolis, n. 3, v. 21, p. 513-518, jul./set. 2012.

GOLD - GLOBAL INITIATIVE FOR CHRONIC OBSTRUCTIVE LUNG DISEASE. Global Strategy for the Diagnoses, management, and prevention of Chronic Obstructive Pulmonary Disease updated 2014. Disponível em: <http://www.goldcopd.org/>. Acesso em: 20 jul. 2014.

NASCIMENTO, E. S. P. et al. Influência de variáveis funcionais e clínicas na qualidade de vida de pacientes com DPOC. ConScientiae Saúde, São Paulo, n. 2, v. 13, p. 234-240, jun. 2014.

NUSSBAUMER-OCHSNER, Y.; RABE, K. F. Systemic manifestations of COPD. Chest, n. 1, v. 139, p. 165-173, jan. 2011.

OLIVEIRA, L. A. et al. Relação do trabalho desenvolvido em testes máximo e submáximo de capacidade de exercício com o grau de obstrução ao fluxo aéreo em indivíduos com Doença Pulmonar Obstrutiva Crônica. Fisioterapia e Pesquisa, São Paulo, n. 1, v. 21, p. 81-86, jan./mar. 2014.

PASQUA, F. et al Use of functional independence measure in rehabilitation of inpatients with respiratory failure. Respiratory Medicine, n. 3, v. 103, p. 471-476, mar. 2009. PROBST, V. S. et al. Reference values for the incremental shuttle walking test. Respiratory Medicine, n. 2, v. 106, p. 243-248, fev. 2012.

RADOSAVLJEVIC, N. et al. Evaluation of functional recovery by motor functional independence measure test of elderly after hip fracture in Serbia. Acta Médica Portuguesa, n. 1, v. 27, p. 88-91, jan./fev. 2014.

SANTOS, D. B.; VIEGAS, C. A. A. Correlação dos graus de obstrução na DPOC com lactato e teste de caminhada de seis minutos. Revista Portuguesa de Pneumologia, Lisboa, n. 1, v. 15, p. 11-25, jan./fev. 2009.

SPOSITO, G. et al. Relações entre o bem-estar subjetivo e a funcionalidade em idosos em seguimento ambulatorial. Revista Brasileira de Fisioterapia, São Carlos, n. 1, v. 14, p. 81-89, jan./fev. 2010.

SPRUIT, M. A. et al. An official american thoracic society/european respiratory society statement: key concepts and advances in pulmonary rehabilitation. American Journal of Respiratory and Critical Care Medicine, Nova York, n. 8, v. 188, p. 13-64, out. 2013.

TALMELLI, L. F. S. et al. Nível de independência funcional e déficit cognitivo em idosos com doença de Alzheimer. Revista da Escola de Enfermagem da USP, São Paulo, n. 4, v. 44, p. 933-939, dez. 2010. 